\title{
DETECCIÓN DE LA DISCRIMINACIÓN HACIA LOS POBRES, «APOROFOBIA»
}

\author{
EVA MARÍA PICADO VALVERDE ${ }^{1}$ \\ ANA BELÉN NIETO LIBRERO ${ }^{2}$ \\ RAQUEL GUZMÁN ORDAZ ${ }^{3}$ \\ AMAIA YURREBASO MACHO ${ }^{4}$ \\ ÁLVARO JÁÑEZ GONZÁLEZ ${ }^{5}$
}

Fecha de recepción: marzo 2019

Fecha de aceptación y versión definitiva: octubre 2019

RESUMEN: El concepto de "aporofobia" nos ayuda a reconocer, identificar y visualizar la discriminación hacia los menos visibles de nuestra sociedad (Cortina, 2000). El rechazo al pobre surge de la construcción de ideas estereotipadas y de los prejuicios hacia las personas excluidas. Aunque el concepto de aporofobia cada vez es más utilizado y su significado es reconocido por profesionales y sociedad, es necesario determinar el conocimiento real que tienen los profesionales sobre el rechazo al pobre. Este estudio pretende configurar la percepción de los trabajadores del ámbito social sobre las victimizaciones sufridas por personas en exclusión social. Tras concluir que éstos identifican diferentes discriminaciones sufridas por sus usuarios, no existen procedimientos para una detección de las discriminaciones y agresiones relacionadas con la aporofobia.

PALABRAS CLAVE: aporofobia; discriminación al pobre; exclusión; prejuicio; estereotipo.

\footnotetext{
1 Universidad de Salamanca. Facultad de Psicología. Correo electrónico: evapicado@usal.es.

2 Universidad de Salamanca. Facultad de Economía y Empresa. Correo electrónico: ananieto@usal.es.

3 Universidad de Salamanca. Facultad de Sociología. Correo electrónico: r.guzman@usal.es.

4 Universidad de Salamanca. Facultad de Psicología. Correo electrónico: amaiay@usal.es.

5 Universidad de Salamanca. Facultad de Relaciones Laborales. Correo electrónico: alvarojg@usal.es.
} 


\section{Detecting discrimination against the poor, "aporophobia"}

ABSTRACT: The concept of "aporophobia" helps us to recognise, identify and conceptualise the discrimination of those who are ignored in our society (Cortina, 2000). Rejection of the poor arises from a construct of stereotypes and prejudice towards socially excluded people. While the concept of aporophobia is increasingly used and its significance is recognised by professional organisations and civil society, it is necessary to determine the real knowledge the professionals have about the rejection of the poor. This study intends to discover how social service workers perceive the victimisations suffered by socially excluded people. Following the discovery that the workers identify different forms of discrimination suffered by their users, it is concluded that no procedures exist that detect discrimination and aggressions related to aporophobia.

KEY WORDS: aporophobia; discrimination against the poor; exclusion; prejudice; stereotype.

\section{CONCEPTUALIZACIÓN DE LA APOROFOBIA}

Actualmente, cualquier artículo científico que haga referencia a un documento del año 1990 puede causar rechazo o desinterés académico, sin embargo, en el caso que nos ocupa implicaría la negación de una realidad. El concepto «aporofobia» fue creado y aceptado a partir del año 1990 por la profesora de ética Adela Cortina, pero no es hasta 2016 cuando su uso se generaliza y es utilizado cada vez más por investigadores y profesionales. En el año 2017, la Fundación BBVA reconoce esta palabra como término del año, motivado por su frecuente aparición en medios de comunicación, hasta el punto de que ese mismo año, la Real Academia de la Lengua decide incorporarla a su diccionario.

Aunque alude a comportamientos y actitudes que se han producido desde hace siglos, según Cortina et al. (2017) era necesario conceptualizarlo en una palabra con el objeto de darle mayor visualización, teniendo claro que todo lo que se hace visible existe, y esta realidad existe.

El término aporofobia (Cortina, 1996) significa rechazo al pobre. Cuando hablamos de rechazo no podemos olvidar el pensamiento, emocionalidad y comportamiento que despierta. Se puede visualizar la imagen de una persona de la que, sin tener dato alguno, solemos incluir elementos de juicio, juicios de valor, que nos ayudarán a crear una imagen de su situación personal, económica e incluso social. El rechazo al pobre no se refiere a 
xenofobia u otros conceptos acuñados con el sufijo de fobia. ¿Reaccionamos de la misma manera al ver a una persona de color si sabemos que es un gran jugador de futbol o ante otra que se encuentra sujetando un carro lleno de cartones y mantas buscando un habitáculo para pernoctar o que se encuentra en un barco recién rescatado en aguas del Mediterráneo? Precisamente esta es la línea distintiva entre el rechazo al pobre y el rechazo al emigrante.

La sociedad ha configurado unos esquemas mentales que le facilitan la justificación del comportamiento para su propia tranquilidad. El esquema cognitivo, la percepción de identificación con la pobreza o falta de recursos, construye distintos mitos con respecto a las personas pobres, que influyen en el comportamiento que se tiene hacia ellos:

- Los pobres son ladrones, los pobres cometen delitos, los pobres son agresivos.

- Los pobres son pobres por su propia culpa, culpables de su situación, sólo quieren dinero, no quieren salir de su situación.

- Los pobres son drogodependientes y alcohólicos.

Según los últimos datos del Instituto Nacional de Estadística (INE) en España hay 22938 personas sin hogar, de las cuales, el 19,3\% corresponde a personas entre 18 y 29 años, entre 30 y 34 años representan el 38,4\%, entre 45 y 64 años son el 38,4\% y los mayores de 64 años suponen el 3,8\%. El grupo mayoritario es el comprendido en la franja de edad entre 30 y 64 años, 11692 personas, las cuales han sufrido victimizaciones del tipo agresión (4774), robo de pertenencias (7228), timos (3312), insultos y amenazas (7652) y agresiones sexuales (657), siendo mucho mayor este comportamiento hacia las mujeres que a los hombres. En relación al consumo de alcohol y drogas, el $62 \%$ de las personas encuestadas no consumían ninguna sustancia y el 29,6\% lo hacía de forma muy moderada y en cuanto a su relación delictiva, hay 4875 personas con antecedentes penitenciarios, reestructurándose la idea errónea sobre la vinculación de las personas «sin hogar» con la drogodependencia y la delincuencia.

Según Sánchez Morales (2012) el perfil sociodemográfico de las personas «sin hogar» es principalmente varón, de edad intermedia, sin vinculación afectiva o relación de pareja. Aunque en los últimos años se han sumado otros colectivos como son las mujeres, los jóvenes e incluso familias completas (Tezanos, 2009) además de personas con enfermedad física o mental, con adicciones, personas maltratadas o institucionalizadas (Sánchez Morales, 2010). 


\section{2. ¿CÓMO PODEMOS EXPLICAR ESTA REACCIÓN SOCIAL?}

Festinger (1957) en su teoría de la Disonancia Cognitiva, postuló que el hecho de mantener dos ideas contradictorias, produce en nosotros un estado de incomodidad cognitiva. La autopercepción de incoherencia genera consecuencias negativas en los sujetos, que necesitan reelaborar la situación para huir de ese estado aversivo. Para ello elaboran argumentos racionales que justifican ese pensamiento y por consiguiente su comportamiento. La identificación del pobre como una persona delincuente, «viciosa por el consumo de drogas» o la atribución de la situación de pobreza a una responsabilidad personal, permite tranquilizar a los miembros de la sociedad de la culpabilidad, en cuanto a su posible ayuda o cambio de situación. Lo habitual de las personas es que exista consonancia entre nuestras opiniones y actitudes, entre lo que sabemos o creemos y lo que hacemos (Festinger, 1957). Pero en determinadas circunstancias, esa congruencia o consonancia se rompe, bien porque no controlamos toda la información que nos llega sobre un hecho o circunstancia, o porque ésta sea contradictoria con la preexistente. Cuando esto ocurre, lo habitual es tratar de racionalizar estas inconsistencias generado nuevos discursos que la mitiguen o eliminar aquellas informaciones que lo sustentan. La disonancia es psicológicamente incómoda, actúa como factor de motivación que lleva al sujeto, en aras de recuperar su propia tranquilidad, a evitar situaciones e informaciones que puedan mantenerla o amentarla.

También puede ser explicado desde el comportamiento intergrupal, Turner et al. (1982) definen el grupo social como un conjunto de personas que se identifican con esta categoría y están dispuestas a actuar de acuerdo a las normas de la misma «Colección de individuos que se perciben a sí mismos como miembros de la misma categoría social, que compartan sentimientos relacionados con esa misma definición común, y que logran un grado de consenso acerca de la evaluación de su grupo y su pertenencia a él» (Tajfel y Turner, 1979).

La categorización o percepción de pertenencia grupal genera en nosotros una percepción de homogeneidad entre los miembros del grupo en nuestras actitudes y conductas, facilitando la distintividad social con respecto a otros grupos. Lo habitual es tomar al grupo como referencia para construir tu propia identidad, asumiendo sus valores y normas con las que regulamos nuestra vida en el grupo (Turner, 1982). Esta identidad social tiene una dimensión externa basada en la relación con otros grupos, y otra interna, basada en la conciencia de nuestra pertenencia al grupo y de lo que el grupo representa para nosotros (Turner, 1987). 
Como grupo, además de mantener interacciones dentro de nuestro grupo, también mantenemos relaciones intergrupales, siendo estas últimas las que mayor impacto generan en nosotros. Podemos afirmar que los grupos son como herramientas cognitivas que permiten segmentar, clasificar y ordenar el medio social, permitiéndonos llevar a cabo distintas formas de acción social. Los grupos nos ofrecen una identificación social de nosotros mismos, relacionada y comparada: somos iguales o diferentes a, mejores o peores que...) y facilitan la orientación, ya que crean y definen el lugar que ocupamos como personas en la sociedad.

Si aplicamos esto al concepto objeto de estudio, tenemos que considerar dos grupos diferenciados, el grupo de «los sin techo» y el grupo de los «con techo». En la distinción de estos dos grupos se incluyen un conjunto de categorías que confluyen una realidad diferenciada en ambos, atribuimos características de los «sin techo» con aquellos esquemas mentales más relacionados a los mitos expuestos anteriormente sobre los pobres.

La peculiaridad de la aporofobia, desde el punto de vista de la criminología, en relación a otros comportamientos delictivos o antisociales, se traduce en las siguientes consideraciones:

- Representación de la víctima: no es un ataque personal a la víctima, sino a lo que representa en su grupo de pertenencia. La respuesta discriminatoria no va dirigida a la persona sino a su representación social, la imagen del «pobre», la deshumanización y cosificación de su significado. Lo que Sheriff (1948) explicaba como el comportamiento realizado por uno o más actores hacia otros actores, basado en la identificación de sí mismo o la identificación de los otros como pertenecientes a otras categorías sociales.

- La intencionalidad es manifestar el daño al grupo al que pertenece la víctima. La motivación principal es rechazar a todo lo que representa el «otro» grupo.

- No es un delito provocado por un sentimiento, sino un comportamiento generado por un conjunto de elementos estereotipados y prejuiciosos.

Cuando nos referimos a los comportamientos discriminatorios estamos hablando de la intolerancia. El artículo 2 de la Declaración Universal de los Derechos Humanos (1948) defiende el derecho de todas las personas sin distinción alguna de raza, color, sexo, idioma, religión, opinión política o de cualquier otra índole de origen nacional o social, posición económica, nacimiento o cualquier otra condición. Por este motivo la aporofobia es la expresión más grave de discriminación al pobre y de violación de los derechos humanos. Según Giddens (1991) la discriminación es el resultado 
conductual de un complejo sistema de relaciones entre los grupos sociales, que limitan o niegan el acceso de oportunidades a un grupo social y sus miembros.

El germen de un comportamiento discriminatorio está configurado por el estereotipo y el prejuicio. Los estereotipos son considerados como la forma de categorizar a las personas para entender la realidad en la que vivimos. Este proceso fue entendido por Allport (1954) como inevitable y según teorías más actuales es considerado como un proceso automático, siendo estas las percepciones adheridas a una persona por la pertenencia a un grupo (Tajfel y Turner, 1979). Según, Oakes et al. (1994) son juicios basados en categorizaciones fluidas que dependen ampliamente del contexto comparativo en que se generan.

Desde la perspectiva más psicológica, el prejuicio ha sido definido como una actitud constituida por un componente cognitivo, un componente afectivo y un comportamiento conductual (Krech, Crutchfield y Ballachey, 1962; McGuire, 1989; Rosenberg y Hovland, 1960; Himmelfarb y Eagly, 1974).

La integración de la perspectiva sociológica, haciendo hincapié desde la explicación de las relaciones intergrupales, ha desencadenado el énfasis del nivel individual y grupal para explicar el prejuicio. Eagly y Diekman (2005) consideran este concepto como un mecanismo intrapsíquico cuyo fin es mantener un estatus y un rol en las diferencias intergrupales, los individuos que se desvían de su rol grupal tradicional despiertan reacciones negativas en el resto de los miembros del grupo.

Dovidio et al. (2010) definen el prejuicio como una actitud individual hacia un grupo y sus miembros, creando o manteniendo relaciones jerárquicas de estatus entre los grupos.

El prejuicio es una actitud negativa hacia alguien y el estereotipo es una creencia generalizada sobre los atributos de un grupo que pueden generar un prejuicio. Se distinguen estos dos conceptos según su funcionalidad, ya que, el estereotipo tiene un carácter descriptivo, mientras que, el prejuicio es evaluativo y emocional (Vicente, 1999).

Según Brown (1995), el prejuicio es la tendencia a poseer actitudes sociales o creencias cognitivas derogatorias, expresar afecto negativo o presentar conductas discriminatorias u hostiles hacia miembros de un grupo por su pertenencia a un grupo concreto.

En la actualidad el prejuicio se explica fundamentalmente desde una perspectiva afectiva, relacionando la cognitiva con el estereotipo o estereotipa, y la conductual con la discriminación, de la misma forma se hace una distinción tipológica del prejuicio como directo y sutil. El prejuicio forma parte de la condición humana. 
Las teorías sobre discriminación y prejuicio pretenden predecir el comportamiento, relacionando el nivel de prejuicio, con el comportamiento manifestado en el exogrupo. Pettigrew y Meertens (1995) y Leyens et al, (2000, 2001) proponen que el proceso de la construcción de la identidad grupal se corresponde a la atribución que hacen los sujetos en función a los grados de humanidad que se da a los otros, de tal forma que atribuimos mayor humanidad a los miembros de nuestro grupo y restamos este elemento a los no pertenecientes al mismo.

El elemento afectivo que explica la manifestación del prejuicio y la discriminación, son la emoción negativa del odio, la rabia y/o la hostilidad. De la misma forma que las emociones son elementos esenciales de nuestra vida y sin ellas no podríamos vivir, estas emociones están condicionadas por la valoración automática de nuestra percepción en las diferentes situaciones. Según Scherer (2001), la evaluación automática activa las diferentes emociones según diferentes premisas:

- Objeto o persona relevante, en nuestro caso el pobre en sí mismo es identificado como persona relevante dentro de los esquemas mentales configurados dentro del grupo de «los pobres», categorizado con las características negativas anteriormente expuestas.

- Implicación del significado "grupo de los pobres» en la vida del grupo propio. Un ejemplo claro es la implicación de la pobreza en el propio grupo, al pensar que para mejorar la vida del grupo de los pobres, el grupo polarizado tiene que trabajar más

- Las habilidades de afrontamiento se manifiestan mostrando mayor rechazo para extinguir o hacer desaparecer el grupo identificado como problema.

- La significación normativa, entendiendo que mostrar animadversión hacia el grupo diferente no rompe ninguna norma social e incluso pensar lo contrario incrementaría la percepción de injusticia social.

Estos condicionantes desencadenan una emoción negativa denominada odio que explica el comportamiento discriminatorio. En realidad el odio es un tipo dentro de la emoción básica denominada como ira (Bisquerra, 2009; Frijda, 1986; Izard, 1977; Plutchik, 1980; Weiner, 1986). Según Potegal, Stemmler y Spielberg (2010) la ira es una emoción con elementos subjetivos, siendo variable la intensidad, los antecedentes condicionantes que explican dicha emoción están relacionados con la percepción que se tengan de ciertas situaciones, cuando percibimos injusticia, ataque personal y limitación de nuestros objetivos o metas por otras personas entre otras. 


\section{OBJETIVOS DE LA INVESTIGACIÓN}

El objetivo de este trabajo es analizar la percepción de la aporofobia por los profesionales de los servicios sociales, tanto de la administración pública, como de las organizaciones del tercer sector, que prestan sus servicios a aquellas personas que se encuentran en situación de exclusión social. El análisis de la percepción de los profesionales en torno a la discriminación al pobre es relevante para identificar, si los profesionales referentes en la atención con estas personas tienen interiorizado la detección de este problema en su praxis diario, o por otra parte trabajan con protocolos donde se establece la detección de la aporofobia y la posible actuación a seguir una vez detectada.

\section{METODOLOGÍA}

Para elaborarlo se solicitó la colaboración de diferentes organizaciones sin ánimo de lucro y técnicos de servicios sociales de las diputaciones de las provincias de Salamanca y Zamora. En todos los recursos seleccionados, los profesionales realizan una atención directa con las personas en exclusión social.

Los datos fueron recogidos mediante un cuestionario estructurado ad hoc vía online. Este estudio exploratorio serviría para la elaboración de un protocolo estandarizado para los profesionales que les facilitaría la valoración del riesgo de sus usuarios en los casos de aporofobia.

La participación de la muestra fue voluntaria y anónima, respondiendo a este cuestionario 43 profesionales. El diseño del cuestionario se ha llevado a cabo teniendo en cuenta la necesidad de recabar datos, que pudieran ofrecernos la información más objetiva y adecuada para diseñar una configuración sobre la percepción de los profesionales, en cuanto a las conductas discriminatorias sobre el rechazo a los pobres y el perfil, tanto de los técnicos, como de los usuarios. Para ello distinguimos las diferentes preguntas a contestar por los profesionales en tres bloques: categoría profesional e identificación de las conductas discriminatorias, tipología del recurso donde trabajan y victimizaciones sufridas por parte de los usuarios. En el cuestionario se utilizaron preguntas cerradas relacionadas con la categoría profesional y el desempeño de su actividad laboral, el tipo de recurso en el que trabaja según las diferentes tipologías de recursos asistenciales, el tipo de victimizaciones detectadas en sus usuarios, los posibles agresores de sus 
usuarios, los tipos de discriminación sufrida las personas con las que trabaja y la motivación presentada por éstos para no formalizar denuncia, así como las actuaciones que realizan los profesionales ante estas situaciones. El diseño de investigación planteado en el estudio es transversal, ya que los datos se han recogido en el único momento temporal planificado en el plan de investigación. La metodología empleada es no experimental, ya que se pretenden describir científicamente las variables analizadas en el grupo de estudio. Se analizan los datos obtenidos de la muestra mediante el programa IBM SPSS Statistics 21.

\section{RESULTADOS}

Tras el análisis de los resultados se concluye que de los 43 profesionales que respondieron al cuestionario, el 39,5\% son profesionales que intervienen con usuarios en un centro de día, el 16,3\% realizan su labor de atención en centros de atención considerados como recursos de primer nivel, es decir, recursos de primera atención a la población, el 14\% realizan su trabajo con las personas en la calle, el 22\% realizan su intervención de forma equitativa en centros residenciales específicos para personas en exclusión y en diversos recursos que no coincidían con las categorías previstas en el cuestionario, mientras que el 7\% restante trabaja en prisión con personas que, aunque en el momento actual residían en un centro penitenciario, cumplían las características de encontrarse en exclusión social.

En cuanto a la categoría profesional de los trabajadores, tal y como refleja el gráfico 1, destaca principalmente la formación en trabajo social, educación y psicología, lo que demuestra la alta cualificación formativa y experta en la intervención comunitaria. 


\section{GRÁFICO 1. CUALIFICACIÓN PROFESIONAL DE LA MUESTRA}

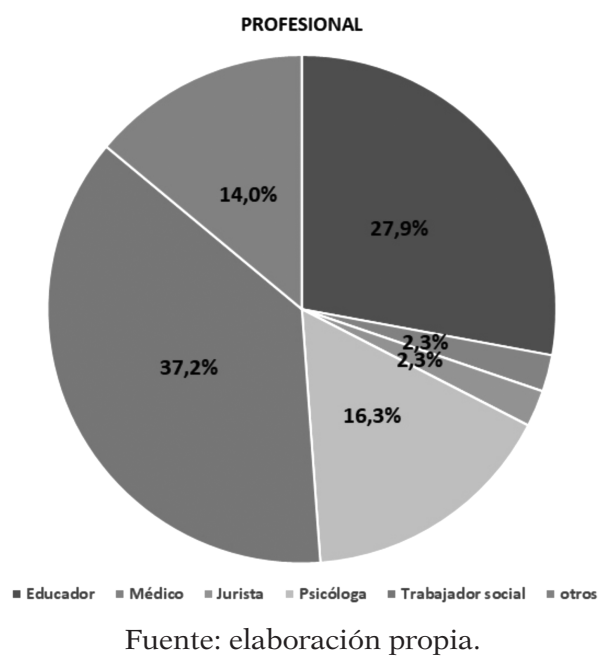

Respecto al número de usuarios atendidos anualmente por los respectivos recursos, prevalecen aquellos que lo hacen con hasta 100 usuarios, como muestra el gráfico 2, siendo muy alto el volumen de atención en los mismos.

\section{GRÁFICO 2. PERSONAS ATENDIDAS EN LOS DIFERENTES RECURSOS}

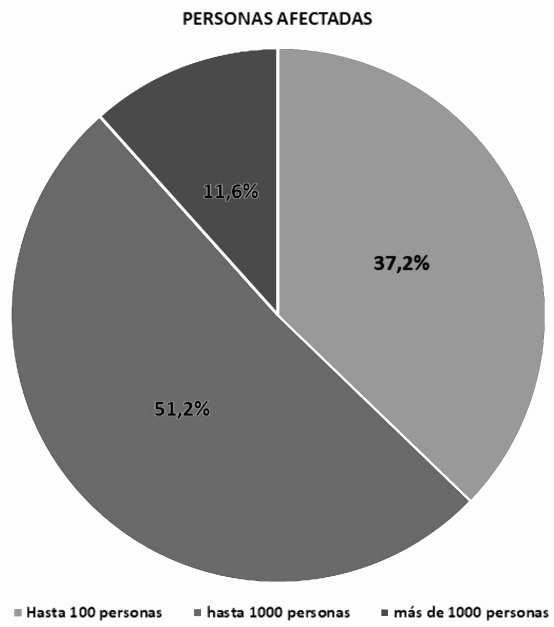

Fuente: elaboración propia. 
En cuanto al predomino de mujeres u hombres entre los recursos de atención, se aprecian diferencias según la tipología del recurso, siendo mayor el porcentaje de mujeres frente al de hombres atendidas en los servicios básicos de atención ( $80 \%$ mujeres), frente al de mayor porcentaje de hombres en la vivienda temporal ( $80 \%$ hombres) y centro de día ( $70 \%$ hombres). La diferenciación de género es muy importante, ya que las mujeres pueden sufrir victimizaciones relacionadas con la discriminación y agresiones sexuales, encontrándose con una doble vulnerabilidad.

En relación a los tipos de victimizaciones sufridas por los usuarios, atendiendo a la percepción de los profesionales, son las agresiones, amenazas, e insultos, y en el 18\% de los casos sufren todas las victimizaciones a lo largo de su vida. Los profesionales perciben que el 2,3\% de los usuarios reconocen un trato discriminatorio, como cuando no se les permite el acceso en un establecimiento.

Los profesionales encuestados consideran que los agresores de los usuarios son principalmente su propia red social, la pareja, los grupos de iguales consumidores de drogas, los recursos de atención, los diferentes sistemas estructurados y el propio entorno, lo que pone de manifiesto la falta de distinción entre las diferentes problemáticas relacionadas con la violencia y la discriminación, como puede ser violencia de género, aporofobia, violencia doméstica etc.

Según los datos aportados por los profesionales, no realizan una distinción ordenada entre las diferentes victimizaciones sufridas, por ejemplo, por agresor o motivación. Al preguntarles sobre las diferentes discriminaciones sufridas por sus usuarios, los profesionales aclaran las diferentes conductas:

- Burlas, insultos.

- Robos.

- Trato vejatorio, desvalorización y falta de respeto.

- Estigmatización por su condición de emigrante, drogodependiente y recluso, además de la patología psiquiátrica.

Según los profesionales, los motivos que tienen estas personas para no denunciar son la normalización de la situación en la que viven, el miedo, la indefensión aprendida y la desconfianza en el sistema judicial. Los trabajadores de los diferentes recursos consideran que su asesoramiento y motivación con éstos para iniciar el procedimiento de denuncia, no está protocolizada, ni está establecida la idoneidad de la resolución de dicha situación. Estos resultados coinciden con el estudio realizado por la Oficina de las Instituciones Democráticas y Derechos Humanos (ODHIR) de la Organización para la Seguridad y la Cooperación en Europa (OSCE) cuyo objetivo era analizar 
las motivaciones principales de la decisión de no denunciar por las víctimas de delitos de odio, siendo las motivaciones más destacadas:

- Temor a nuevas victimizaciones.

- Sentimiento de humillación y vergüenza.

- Percepción de inseguridad al procedimiento de denuncia.

- Desconfianza en el sistema policial y de justicia.

- Falta de identificación propia de la víctima.

- Miedo a las consecuencias de la interposición de la denuncia.

Analizando la cifra oficial, reflejada en el informe del 2016 del Ministerio de Interior, podemos concluir que Castilla y León, no es el territorio de mayor prevalencia de incidentes de aporofobia registrados, señalándose dos en nuestra comunidad y uno de ellos en la provincia de Salamanca.

\section{CONCLUSIONES Y PROSPECTIVA}

Tras los resultados obtenidos, se concluye que los profesionales identifican episodios de violencia que se ejerce a sus usuarios, pero no se discrimina su tipología, ni los agresores aun reconociéndose que acontecen de forma habitual. Con este estudio inicial, se puede predecir la necesidad de incluir cuestiones relacionadas con la forma de afrontar, tanto los usuarios, como los profesionales, las situaciones identificadas como aporofobia.

El reto para la eliminación de la aporofobia, tras el análisis de la situación según la percepción de los profesionales, consiste en que es necesario identificar con detalle las victimizaciones discriminatorias sufridas por los usuarios de los diferentes recursos asistenciales del ámbito social. Proveer de formación a los trabajadores del ámbito social sobre comportamientos discriminatorios y de odio para aprender a detectar estas situaciones, además de construir protocolos, procedimientos e instrumentos de gestión de riesgo para dotar de herramientas eficaces a los profesionales, que les generen seguridad en su labor de detección e intervención con personas en exclusión social.

Otro objetivo, a conseguir en el estudio de la aporofobia, es la inclusión de la perspectiva de género como parte de la intervención de la prevención y eliminación contra los delitos de odio. Existen diferencias de victimización y de riesgo en función del género, los prejuicios de género pueden ser un factor precipitante o de motivación de esta delincuencia de discriminación. Hay delitos que son más frecuentes en los hombres y otros más entre mujeres, por ejemplo, la delincuencia sexual es más prevalente en mujeres, mientras que las agresiones físicas son más contra los hombres. 


\section{REFERENCIAS}

Allport, G. (1954). The nature of prejudice. Reading, USA: Addison-Wesley Publishing Company.

Andrade, M. (2008). ¿Qué es la aporofobia? un análisis conceptual sobre prejuicios, estereotipos y discriminación hacia los pobres. Agenda social, 2(3), 117-139.

Bisquerra, R. (2009). Psicopedagogía de las emociones. Madrid, España: Síntesis.

Brown, R. (1995). Prejudice: Its social psychology. Oxford, UK: Blackwell.

Cortina, A. (1996). Ética. Madrid: Santillana

Cortina, A. (2017). Aporofobia, el rechazo al pobre. Un desafío para la democracia. Barcelona, España: Paidós.

Dovidio, J., Hewstone, M., Glick, P., y Esses, V. (2010). Prejudice, Stereotyping and Discrimination: Theoretical and Empirical Overview. En J. Dovidio, M. Hewstone, P. Glick y V. Esses (Eds.), The SAGE Handbook of Prejudice, Stereotyping and Discrimination (pp. 3-28). London, UK: SAGE Publications Ltd. https://doi. org/10.4135/9781446200919.

Eagly, A., y Diekman, A. (2005). What is the problem? Prejudice as an attitude-incontext. En J. Dovidio, P. Glick y L. Rudman (Eds.), On the nature of prejudice: Fifty years after Allport (pp. 19-35). Malden, USA: Blackwell.

Festinger, L. (1975). Teoría de la disonancia cognoscitiva. Madrid, España: Instituto de Estudios Políticos.

Frijda, N. (1986). The emotions. Cambridge, UK: Cambridge University Press.

Giddens, A. (1991). Sociología. Madrid, España: Alianza Editorial.

Himmelfarb, S., y Eagly, A. (1974). Readings in attitude change. Oxford, UK: John Wiley \& Sons.

Izard, C. (1977). Human emotions. Nueva York, USA: Plenum Press.

Krech, D., Crutchfield, R., y Ballachey, R. (1962). Individual in society. New York, USA: McGraw-Hill.

Leyens, J., Paladino, P., y Rodríguez, R. (2000). The emotional side of prejudice: The attribution of secondary emotions to ingroups and outgroups. Personality and Social Psychology Review, 4(2), 186-197. https://doi.org/10.1207/S15327957PSPR0402_06.

Leyens, J., Rodríguez, A., Rodríguez, R., Gaunt, R., Paladino, P., Vaes, J., y Demoulin, S. (2001). Psychological essentialism and the differential attribution of uniquely human emotions to ingroups and outgroups. European Journal of Social Psychology, 31(4), 395-411. https://doi.org/10.1002/ejsp.50.

McGuire, W. J. (1989). The structure of individual attitudes and attitude systems. En A. Pratkanis, S. Breckler y A. Greenwald (eds.), The third Ohio State University Vol. on attitudes and persuasion. Attitude structure and function (pp. 37-69). Hillsdale, USA: Lawrence Erlbaum Associates, Inc.

Ministerio del Interior (2016). Informe sobre la evolución de los incidentes relacionados con los Delitos de Odio en España. Disponible en http://www.interior.gob. es/documents/10180/5791067/ESTUDIO+INCIDENTES+DELITOS+DE+OD IO+2016.pdf/c5ef4121-ae02-4368-ac1b-ce5cc7e731c2.

Muñoz, M., Vazquez, C., y Vázquez, J. (2003). Los límites de la exclusión social. Madrid, España: Ediciones Témpora. 
Naciones Unidas (1948). Declaración Universal de los Derechos Humanos. Recuperado de: https://www.derechoshumanos.net/normativa/normas/1948-DeclaracionUniversal.htm.

Oakes, P., Haslam, A., y Turner, J. (1994). Stereotyping and social reality. Oxford: Blackwell.

Organización para la Seguridad y la Cooperación en Europa (OSCE). La lucha contra los Delitos de Odio en la Región Osce. Disponible en: https://plataformaciudadanacontralaislamofobia.files.wordpress.com/2016/01/delitosodiooscenumero5.pdf.

Pettigrew, T., y Meertens, R. (1995). Subtle and blatant prejudice in Western Europe. European Journal of Social Psychology 25(1), 57-75. https://doi.org/10.1002/ ejsp.2420250106.

Plutchik, R. (1980). Emotion: A psychoevolutionary synthesis. Nueva York, USA: Harper and Row.

Potegal, M., Stemmler, G., y Spielberger, Ch. (2010). International Handbook of Anger: Biological, Psychological, and Social Processes. Nueva York, USA: Springer.

Rosenberg, M., y Hovland, C. (1960). Cognitive, affective, and behavioral components of attitude. En M. Rosenberg, C. Hovland, W. McGuire, R. Abelson y J. Brehm (eds). Attitude Organization and change: An analysis of consistency among attitude components. New Haven, USA: Yale University Press.

Sánchez, M. (2010). Las personas «sin hogar» en España. Revista Española de Sociología, (14), 21-42.

Sánchez, M. (2012). En los límites de la exclusión social: las personas sin hogar en España. Revista de Ciencias Sociales, 7 (2), 307-324.

Scherer, K. (2001). Appraisal considered as a process of multilevel sequential checking. En K. Scherer, A. Schorr y T. Johnstone (Eds.), Appraisal processes in emotion: Theory, methods, research (pp. 92-120). Nueva York, USA: Oxford University Press.

Sherif, M. (1948). An outline of social psychology. New York, USA: Harper and Row.

Smith, V. (2006). La psicología social de las relaciones intergrupales: modelos e hipótesis. Actualidades en psicología, 20(102), 45-71. DOI: https://doi.org/10.15517/ ap.v20i107.37.

Tajfel, H. y Turner, J. (1979). An integrative theory of intergroup conflict. En W. Austin y S. Worchel (Eds). The social psychology of intergroup relation (pp. 33-47). Monterey, USA: Brooks/Cole.

Tezanos, J. (2009). Juventud y exclusión social. Décimo Foro sobre Tendencias Sociales. Madrid, España: Editorial Sistema.

Turner, J. (1982). Towards a cognitive redefinition of the social group. En H. Tajfel (ed.): Social identity and intergroup relations. Cambridge, UK: Cambridge University Press.

Turner, J., Hogg, M., Oakes, P., Reicher, S., y Wetherell, M. (1987). Rediscovering the social group: A self-categorization theory. Cambridge, USA: Basil Blackwell.

Vicente, F. (1999). Construcción Psicológica de la Identidad Regional. Tópicos y Estereotipos en el Proceso de Socialización: el referente a Extremadura. Badajoz, España: Diputación Provincial de Badajoz.

Weiner, B. (1986). An attributional theory of motivation and emotion. Nueva York, USA: Springer-Verlag. 